\title{
Stochastic lot sizing problem with controllable processing times
}

\author{
Esra Koca, Hande Yaman, M. Selim Aktürk* \\ Department of Industrial Engineering, Bilkent University, Ankara 06800 Turkey
}

\section{A R T I C L E I N F O}

\section{Article history:}

Received 19 January 2014

Accepted 6 November 2014

Available online 24 November 2014

Keywords:

Stochastic lot sizing

Controllable processing times

Second order cone programming

\begin{abstract}
A B S T R A C T
In this study, we consider the stochastic capacitated lot sizing problem with controllable processing times where processing times can be reduced in return for extra compression cost. We assume that the compression cost function is a convex function as it may reflect increasing marginal costs of larger reductions and may be more appropriate when the resource life, energy consumption or carbon emission are taken into consideration. We consider this problem under static uncertainty strategy and $\alpha$ service level constraints. We first introduce a nonlinear mixed integer programming formulation of the problem, and use the recent advances in second order cone programming to strengthen it and then solve by a commercial solver. Our computational experiments show that taking the processing times as constant may lead to more costly production plans, and the value of controllable processing times becomes more evident for a stochastic environment with a limited capacity. Moreover, we observe that controllable processing times increase the solution flexibility and provide a better solution in most of the problem instances, although the largest improvements are obtained when setup costs are high and the system has medium sized capacities.
\end{abstract}

(c) 2014 Elsevier Ltd. All rights reserved.

\section{Introduction}

In this paper, we consider the lot sizing problem with controllable processing times where demand follows a stochastic process and processing times of jobs can be controlled in return for extra cost (compression cost). Processing time of a job can be controlled (and reduced) by changing the machine speed, allocating extra manpower, subcontracting, overloading, consuming additional money or energy. Although these options are available in many real life production and inventory systems, in the traditional studies on the lot sizing problem, processing times of jobs are assumed as constant.

Since the seminal paper of Wagner and Whitin [40], the lot sizing problem and its extensions have been studied widely in the literature (see $[13,23]$ for a detailed review on the variants of the lot sizing problem). In the classical lot sizing problem, it is assumed that the demand of each period is known with certainty although this is not the case for most of the production and inventory systems and approximating the demand precisely may be very difficult. In the stochastic lot sizing problem, this assumption is relaxed but the probability distribution of the demand is assumed as known.

As reducing processing time of a job is equivalent to increasing production capacity, subcontracting, overloading or capacity acquisition

\footnotetext{
This manuscript was processed by Associate Editor Geunes.

* Corresponding author.

E-mail addresses: ekoca@bilkent.edu.tr (E. Koca), hyaman@bilkent.edu.tr (H. Yaman), akturk@bilkent.edu.tr (M. Selim Aktürk).

can be seen as special cases of the controllable processing times. There are studies in the literature that consider the lot sizing problem with subcontracting (or outsourcing) $[3,10,18]$ or capacity acquisition (or expansion) $[1,17,22]$. However, in all these studies costs of these options are assumed as linear or concave. This assumption makes it possible to extend the classical extreme point or optimal solution properties for these cases. In our study, we assume that the compression cost is a convex function of the compression amount.

Controllable processing times are well studied in the context of scheduling. Earlier studies on this subject assume linear compression costs as adding nonlinear terms to the objective (total cost) function may make the problem more difficult [14]. However, as it is stated in recent studies, reducing processing times gets harder (and more expensive) as the compression amount increases in many applications [14,2]. For example, by increasing machine speed, processing times can be reduced, but this also decreases life of the tool and an additional tooling cost is incurred. Moreover, increasing the machine speed may also increase the energy consumption of the facility. Another example is a transportation system in which trucks may be overloaded or their speeds could be increased in return for extra cost due to increasing fuel consumption or limiting the carbon emission. Thus, considering a convex compression cost function is realistic since a convex function represents increasing marginal costs and may limit higher usage of the resource due to environmental issues.

In our study, we consider the following convex compression cost function for period $t: \gamma_{t}\left(k_{t}\right)=\kappa_{t}\left(k_{t}\right)^{a / b}$ where $k_{t}>0$ is the total compression amount in period $t, \kappa_{t} \geq 0$ and $a \geq b>0, a, b \in Z_{+}$. Note that, for $a>b$ and $\kappa_{t}>0, \gamma_{t}$ is strictly convex. This function can 
represent increasing marginal cost of compressing processing times in larger amounts. Moreover, this function can be related to a (convex) resource consumption function $[25,28]$. Suppose that one additional unit of the resource costs $\kappa_{t}$ and for compressing the processing time by $k_{t}$ units, additional $k_{t}^{a / b}$ units of resource should be allocated. Thus, in this context, compression cost represents resource consumption cost and the resource may be a continuous nonrenewable resource such as energy, fuel or catalyzer. With the recent advances in convex programming techniques, many commercial solvers (like IBM ILOG CPLEX) can now solve second-order cone programs (SOCP). In this study, we make use of this technique and formulate the problem as SOCP so that it can be solved by a commercial solver.

The contributions of this paper are threefold:

- To the best of our knowledge, this is the first study that considers the stochastic lot sizing problem with controllable processing times. Although this option is applicable to many real life systems, the processing times are assumed as constant in the existing literature on lot sizing problems.

- The inclusion of a nonlinear compression cost function complicates the problem formulation significantly. Therefore, we utilize the recent advances in second-order cone programming to alleviate this difficulty, so that the proposed conic formulations could be solved by a commercial solver in a reasonable computation time instead of relying on a heuristic approach.

- Since assuming fixed processing times unnecessarily limits the solution flexibility, we conduct an extensive computational experiments to identify the situations where controlling the processing times improves the overall production cost substantially.

The rest of the paper is organized as follows. In the next section, we briefly review the related literature. In Section 3, we formulate the problem and in Section 4, we strengthen the formulation using the second-order conic strengthening. In Section 5, we present the results of our computational experiments. We first compare alternative conic formulations presented in Section 5, afterwards we investigate the impact of controllable processing times on production costs. In Section 6, conclusions and future research directions are discussed.

\section{Literature review}

Here, we first review the studies on stochastic lot sizing problems. Silver [30] suggests a heuristic solution procedure for solving the stochastic lot sizing problem. Laserre et al. [16] consider the stochastic capacitated lot sizing problem with inventory bounds and chance constraints on inventory. They show that solving this problem is equivalent to solving a deterministic lot sizing problem. Bookbinder and Tan [5] study the stochastic uncapacitated lot sizing problem with $\alpha$-service level constraints under three different strategies (static uncertainty, dynamic uncertainty and staticdynamic uncertainty). Service level $\alpha$ represents the probability that inventory will not be negative. In other words, it means that with probability $\alpha$, the demand of any period will be satisfied on time. Under the static uncertainty decision rule, which is the strategy that will be used in our study, all the decisions (production and inventory decisions) are taken at the beginning of the planning horizon (frozen schedule). The authors formulate the problem and show that their model is equivalent to the deterministic problem by showing the correspondence between the terms of these two formulations.

Service level constraints are mostly used in place of shortage or backlogging costs in the stochastic lot sizing problems. Since shortages may lead to loss of customer goodwill or delays on the other parts of the system, it may be hard to estimate the backlogging or shortage costs in the real life production and inventory systems. Rather than considering the backlogging cost as a part of the total cost function, a specified level of service (in terms of availability of stock) can be assured by service level constraints and when the desired service level is high, backlogging costs can be omitted. This situation makes the usage of service level constraints more popular in the real life systems $[5,19,6]$. A detailed investigation of different service level constraints can be found in Chen and Krass [6].

Vargas [38] studies (the uncapacitated version of) the problem of Bookbinder and Tan [5] but rather than using service level constraints he assumes that there is a penalty cost for backlogging, the cost components are time varying and there is a fixed lead time. He develops a stochastic dynamic programming algorithm, which is tractable when the demand follows a normal distribution. Sox [31] studies the uncapacitated lot sizing problem with random demand and non-stationary costs. He assumes that the distribution of demand is known for each period and considers the staticuncertainty model, but uses penalty costs instead of service level constraints. He formulates the problem as an MIP with nonlinear objective (cost) function and develops an algorithm that resembles the Wagner-Whitin algorithm.

In the static-dynamic uncertainty strategy of Bookbinder and Tan [5], the replenishment periods are determined first, and then replenishment amounts are decided at the beginning of these periods. They also suggest a heuristic two-stage solution method for solving this problem. Tarim and Kingsman [32] consider the same problem and formulate it as MIP. Moreover, Özen et al. [20] develop a non-polynomial dynamic programming algorithm to solve the same problem. Recently, Tunç et al. [36] reformulate the problem as MIP by using alternative decision variables and Rossi et al. [24] propose an MIP formulation based on the piecewise linear approximation of the total cost function, for different variants of this problem.

In the dynamic uncertainty strategy, production decision for any period is made at the beginning of that period. Dynamic and static-dynamic strategies are criticized due to the system nervousness they cause; supply chain coordination may be problematic under these strategies since the production decision for each period is not known until the beginning of the period [34,35].

There are studies in the literature, in which instead of $\alpha$ service level, fill rate criterion ( $\beta$ service level) is used. Fill rate can be defined as the proportion of demand that is filled from available stock on hand. Thus, this measure also includes information about the backordering size. Tempelmeier [33] proposed a heuristic approach to solve the multi-item capacitated stochastic lot-sizing problem under fill rate constraint. Helber et al. [10] consider the multi-item stochastic capacitated lot sizing problem under a new service level measure, called as $\delta$-service-level. This service level reflects both the size of the backorders and waiting time of the customers and can be defined as the expected percentage of the maximum possible demand-weighted waiting time that a customer is protected against. The authors assume that the cost components are time invariant and there is an overtime choice with linear costs for each period. They develop a nonlinear model and approximate it by two different linear models.

There are also studies in the literature that consider the lot sizing problem with production rate decisions [41] or with quadratic quality loss functions [12]. However, they consider the problem under an infinite horizon assumption.

Another topic related to our study is controllable processing times, which is well studied in the context of scheduling. One of the earliest studies on scheduling with controllable processing times is conducted by Vickson [39]. Kayan and Aktürk [14] and Aktürk et al. [2] consider a CNC machine scheduling problem with controllable processing times and convex compression costs. Jansen and Mastrolilli [11] develop approximation schemes, Gürel et al. [9] use an anticipative approach to form an initial solution, Türkcan et al. [37] 
use a linear relaxation based algorithm for the scheduling problem with controllable processing times. Shabtay and Kaspi [25], Shabtay and Kaspi [26] and Shabtay and Steiner [28] study the scheduling problem with convex resource consumption functions. The reader is referred to Shabtay and Steiner [27] for a detailed review on scheduling with controllable processing times.

In this study, we will consider the static uncertainty strategy of Bookbinder and Tan [5]. Formulations given in this paper are similar to theirs; but there are two major differences. First, our system is capacitated and note that even the capacitated deterministic lot sizing problem with varying capacities is NP-Hard. Second, we will assume that the processing times are controllable and compression cost is a convex function. In the next section, a formal definition of the problem and formulations will be given.

\section{Problem definition and formulations}

We consider the stochastic capacitated lot sizing problem with service level constraints and controllable processing times. We assume that the demand of each period is independent from each other and normally distributed with mean $\mu_{t}$ and standard deviation $\sigma_{t}$ for period $t=1, \ldots, T$, where $T$ is the length of the planning horizon. We denote the demand of period $t$ by $d_{t}$. We allow backlogging but assume that all the shortages are satisfied as soon as a supply is available. We restrict this case by using $\alpha$ service level constraints, where $\alpha$ corresponds to the probability of no stock out in a period. We assume that the resource is capacitated and capacity of period $t$ in terms of time units is indicated by $C_{t}$. Processing time of an item is $p_{t}$ time units, but we can reduce (compress) it in return for extra cost (compression cost). The processing time of an item can be reduced by at most $u_{t}\left(<p_{t}\right)$ time units. We assume that all the production decisions are made at the beginning of the planning horizon. The problem is to find a production plan that satisfies the minimum service level constraints and minimizes the total production, compression and inventory costs.

Let $x_{t}$ be the production amount in period $t, y_{t}=1$ if there is a setup in period $t$ and 0 otherwise, and $s_{t}$ be the inventory on hand at the end of period $t$. We define $\gamma_{t}: \mathbb{R}_{+} \rightarrow \mathbb{R}_{+}$as the compression cost function and $k_{t}$ as the total compression amount (reduction in processing time) in period $t$. We assume that $\gamma_{t}$ is a convex function. Let $q_{t}, c_{t}$, and $h_{t}$ be the setup, unit production and inventory holding costs for period $t$, respectively. The problem can be formulated as the following:

$$
\begin{aligned}
& \text { LS-I min } \quad \sum_{t=1}^{T}\left(q_{t} y_{t}+c_{t} x_{t}+h_{t} \mathbb{E}\left[\max \left\{s_{t}, 0\right\}\right]+\gamma_{t}\left(k_{t}\right)\right) \\
& \text { s.t. } \quad s_{t}=\sum_{i=1}^{t} x_{i}-\sum_{i=1}^{t} d_{i}, \quad t=1, \ldots, T, \\
& \operatorname{Pr}\left\{s_{t} \geq 0\right\} \geq \alpha, \quad t=1, \ldots, T, \\
& p_{t} x_{t}-k_{t} \leq C_{t} y_{t}, \quad t=1, \ldots, T, \\
& k_{t} \leq u_{t} x_{t}, \quad t=1, \ldots, T, \\
& x_{t}, k_{t} \geq 0, \quad t=1, \ldots, T, \\
& y_{t} \in\{0,1\}, \quad t=1, \ldots, T .
\end{aligned}
$$

In constraints (2), inventory at the end of each period is expressed. Note that we assume that the initial inventory is zero. If this is not the case, we can easily add $s_{0}$ to the right hand side of constraint (2). The probability expressed in constraint (3) is the probability that no stock-out occurs in period $t$ and this should be greater than or equal to $\alpha$. Constraint (4) is the capacity constraint: if $x_{t}$ units are produced in period $t, p_{t} x_{t}$ time units are necessary for production without any compression, but if this is larger than the capacity $C_{t}$, then we need to reduce the processing times by $k_{t}=C_{t}-p_{t} x_{t}$ in total. Since processing time of a unit cannot be reduced more than $u_{t}$ time units and $x_{t}$ units are produced in period $t$, total compression amount $k_{t}$ should be less than or equal to $u_{t} x_{t}$, and this is ensured by (5).

In our problem, since $d_{t}$ is a random variable (with known distribution), $s_{t}$ is also a random variable. Therefore, from constraint (2), expected inventory at the end of each period can be obtained as $\mathbb{E}\left[s_{t}\right]=\sum_{i=1}^{t} x_{i}-\sum_{i=1}^{t} \mathbb{E}\left[d_{i}\right], t=1, \ldots, T$.

Let $G_{d_{1 t}}$ be the cumulative probability distribution of the cumulative demand up to period $t$, which is denoted by $d_{1 t}=\sum_{i=1}^{t} d_{i}$. Since demand of each period is independent from each other, $d_{1 t}$ is normally distributed with mean $\mu_{1 t}=\sum_{i=1}^{t} \mu_{i}$ and standard deviation $\sigma_{1 t}=\sqrt{\sum_{i=1}^{t} \sigma_{i}^{2}}$. Therefore, we can rewrite the $\alpha$ service level constraint (3) as

$$
\begin{aligned}
& \operatorname{Pr}\left\{S_{t}\right.\geq 0\}=\operatorname{Pr}\left\{\sum_{i=1}^{t} x_{i} \geq \sum_{i=1}^{t} d_{i}\right\}=G_{d_{1 t}}\left(\sum_{i=1}^{t} x_{i}\right) \geq \alpha \\
& \Leftrightarrow \sum_{i=1}^{t} x_{i} \geq G_{d_{1 t}}^{-1}(\alpha) \\
& \Leftrightarrow \sum_{i=1}^{t} x_{i} \geq Z_{\alpha} \sigma_{1 t}+\mu_{1 t},
\end{aligned}
$$

since the inverse cumulative probability of $d_{1 t}$ is $G_{d_{1 t}}^{-1}=Z_{\alpha} \sigma_{1 t}+\mu_{1 t}$ where $Z_{\alpha}$ represents the $\alpha$-quantile of the standard normal distribution [5]. Note that inequality (8) is similar to demand satisfaction constraint of the classical lot sizing problem. Let $\hat{d}_{1}=Z_{\alpha} \sigma_{11}+\mu_{1}$ and $\hat{d}_{t}=Z_{\alpha}\left(\sigma_{1 t}-\sigma_{1(t-1)}\right)+\mu_{t}$ for $t=2, \ldots, T$ be the new demand parameters and suppose $\hat{s}$ denotes the new stock variables. Then, (8) can be expressed as

$$
\begin{aligned}
& \hat{s}_{t-1}+x_{t}=\hat{d}_{t}+\hat{s}_{t}, \quad t=1, \ldots, T \\
& \hat{s}_{0}=0 \\
& \hat{s}_{t} \geq 0, \quad t=1, \ldots, T .
\end{aligned}
$$

Finally, as we assume that $\alpha$ is sufficiently large and shortages are fulfilled as soon as a supply is available, we can approximate the expected total inventory cost as done in Bookbinder and Tan [5]

$$
\begin{aligned}
\sum_{t=1}^{T} h_{t}\left(\mathbb{E}\left[\max \left\{s_{t}, 0\right\}\right]\right) & \approx \sum_{t=1}^{T} h_{t}\left(\sum_{i=1}^{t} x_{i}-\sum_{i=1}^{t} \mathbb{E}\left[d_{i}\right]\right) \\
& =\sum_{t=1}^{T} \bar{h}_{t} x_{t}-h_{t} \mu_{1 t},
\end{aligned}
$$

where $\bar{h}_{t}=\sum_{j=t}^{T} h_{j}$. Let $\bar{c}_{t}=c_{t}+\bar{h}_{t}$, then we can remove the original inventory variables $s_{t}$ from the formulation LS-I and rewrite the objective function (1) as

$$
\sum_{t=1}^{T}\left(q_{t} y_{t}+\bar{c}_{t} x_{t}+\gamma_{t}\left(k_{t}\right)\right)
$$

Now consider the capacitated deterministic lot sizing problem. An interval $[j, l]$ is called a regeneration interval if the initial inventory of period $j$ and the final inventory of period $l$ are zero, and final inventory of any period between $j$ and $l$ is positive. Period $i \in[j, l]$ is called a fractional period if $i$ is a production period but the production amount is not at full capacity level. It is known that when the production and inventory holding cost functions are concave, the lot sizing problem has an optimal solution that is composed of consecutive regeneration intervals and in each of these intervals there exists at most one fractional period. Most of the dynamic programming algorithms developed for variations of the lot sizing problem use variations of this property. The reader is referred to Pochet and Wolsey [21] for more details. As it can be 
observed from the following example, this property does not hold for our problem as our production cost function is not concave.

Example 3.1. Consider the following problem instance: $T=3$, $q_{t}=100, c_{t}=0, h_{t}=1, \kappa_{t}=0.25, C_{t}=20, p_{t}=1, u_{t}=0.5$ for $t=1, \ldots, T$, $a / b=2$ and $\hat{d}=(10,20,10)$. Optimal solution to the problem is $x^{*}=(18,22,0), s^{*}=(8,10,0)$ and $k^{*}=(0,2,0)$ with total cost 219. This solution is composed of one regeneration interval $[1,3]$ and both the production periods in this interval are fractional if the capacity is assumed as $20 /(1-0.5)=40$. Thus, the regeneration interval property of the classical lot sizing problem does not hold for this problem.

Note that the total production and compression cost function for each period has two breakpoints $\hat{C}_{t}=C_{t} / p_{t}$ and $\bar{C}_{t}=C_{t} /\left(p_{t}-u_{t}\right)$. The first segment $\left[0, \hat{C}_{t}\right]$ corresponds to the regular production cost and the second segment $\left[\hat{C}_{t}, \bar{C}_{t}\right]$ corresponds to the cost of production with compression. If $\hat{C}_{t}$ are time dependent then the problem is NP-Hard, since the classical lot sizing problem with arbitrary capacities is a special case of our problem (the case with $u_{t}=0$ for all $t$ ). If $\hat{C}_{t}=C^{1}$ and $\bar{C}_{t}=C^{2}$ for $t=1, \ldots, T$, and $a / b=1$, then the problem is a lot sizing problem with piecewise linear production costs and it can be solved in polynomial time [15]. When $a / b>1$, as the compression cost function is convex and there exist setup costs, it is unlikely to find a polynomial time algorithm for solving the problem since even the uncapacitated lot sizing problem with convex production cost functions and unit setup costs is NP-Hard [8]. Besides, if the compression cost function is piecewise linear and convex, then the total production cost function is also piecewise linear and any formulation for the piecewise linear functions (multiple choice, incremental, convex combination (see, e.g., [7]) or the (pseudo-polynomial time) algorithm of Shaw and Wagelmans [29] can be used. Moreover, as it is stated above, if the breakpoints of the total production cost function is time invariant and the number of breakpoints is fixed, then the problem is polynomially solvable due to the dynamic programming algorithm of Koca et al. [15].

\section{Reformulations}

Now we need to examine the compression cost function $\gamma_{t}($.). There is not much done on this class of lot sizing problems with convex production cost functions, since most of the optimality properties are not valid for this particular case as demonstrated in Example 3.1. Still, as it is shown in this section, the problem we study has some nice structure that we could use to strengthen the formulation.

Assume that compression cost function for period $t$ is given by $\gamma_{t}\left(k_{t}\right)=\kappa_{t} k_{t}^{a / b}$ where $k_{t}>0$ is the total compression amount in period $t, \kappa_{t} \geq 0$ and $a \geq b>0, a, b \in Z_{+}\left(k_{t}=\max \left\{0, p_{t} x_{t}-C_{t}\right\}\right)$. In order to formulate this case, as done in Aktürk et al. [2], we introduce auxiliary variables $r_{t}$, add the following inequalities:

$k_{t}^{a / b} \leq r_{t}, \quad t=1, \ldots, T$,

and replace $\gamma_{t}\left(k_{t}\right)$ with $\kappa_{t} r_{t}$ in the objective function (12). As $b>0$, we can rewrite (13) as

$k_{t}^{a} \leq r_{t}^{b}, \quad t=1, \ldots, T$.

Therefore, we could reformulate the problem as follows:

$$
\begin{aligned}
& \text { LS-II min } \quad \sum_{t=1}^{T}\left(q_{t} y_{t}+\bar{c}_{t} x_{t}+\kappa_{t} r_{t}\right) \\
& \text { s.t. } \quad \hat{s}_{t-1}+x_{t}=\hat{d}_{t}+\hat{s}_{t}, \quad t=1, \ldots, T, \\
& p_{t} x_{t}-k_{t} \leq C_{t} y_{t}, t=1, \ldots, T, \\
& k_{t} \leq u_{t} x_{t}, \quad t=1, \ldots, T, \\
& k_{t}^{a} \leq r_{t}^{b}, \quad t=1, \ldots, T,
\end{aligned}
$$

$$
\begin{aligned}
& \hat{s}_{0}=0, \\
& x_{t}, k_{t}, r_{t}, \hat{s}_{t} \geq 0, \quad t=1, \ldots, T, \\
& y_{t} \in\{0,1\}, \quad t=1, \ldots, T .
\end{aligned}
$$

Moreover, as it is done in Aktürk et al. [2], we can strengthen inequality (14) as

$k_{t}^{a} \leq r_{t}^{b} y_{t}^{a-b}, \quad t=1, \ldots, T$.

Note that if there is no production in period $t$, then $y_{t}=0$ and there will be no need for compression; thus, $k_{t}=0$. On the other hand, if $y_{t}=1$, then inequality (15) reduces to (14).

We will refer to the strengthened version of LS-II (the set of constraints (14) is replaced with the set of constraints (15)) as LS-III. Now we will show that this strengthening gives the convex hull of the set

$S=\left\{(x, k, r, y) \in \mathbb{R}_{+}^{3} \times\{0,1\}: k^{a / b} \leq r, k \leq u x, p x-k \leq C y\right\}$,

where the subscripts are dropped for the ease of presentation. Set $S$ can be seen as a single period relaxation that involves only the production, setup and compression variables associated with a given period. Our hope is that having a strong formulation for set $S$ may be useful in solving the overall problem. The computational results presented in the next section show that this strengthening is indeed useful.

Let

$S^{\prime}=\left\{(x, k, r, y) \in \mathbb{R}_{+}^{4}: k^{a} \leq r^{b} y^{a-b}, k \leq u x, p x-k \leq C y, 0 \leq y \leq 1\right\}$.

Proposition 4.1. $S^{\prime}$ is the convex hull of S, i.e., $\operatorname{conv}(S)=S^{\prime}$.

Proof. First, we will show that $\operatorname{conv}(S) \subseteq S^{\prime}$. Consider $\left(\left(x_{1}, k_{1}, r_{1}\right.\right.$, $\left.y_{1}\right),\left(x_{2}, k_{2}, r_{2}, y_{2}\right) \in S$. Note that if $y_{1}=y_{2}$, then convex combination of these points is in $S \subseteq S^{\prime}$. Thus, suppose that $y_{1}=0$ (and consequently, $x_{1}=k_{1}=0$ ) and $y_{2}=1$. Consider the convex combination of these points:

$$
\begin{aligned}
(x, k, r, y) & =(1-\lambda)\left(0,0, r_{1}, 0\right)+\lambda\left(x_{2}, k_{2}, r_{2}, 1\right) \\
& =\left(\lambda x_{2}, \lambda k_{2},(1-\lambda) r_{1}+\lambda r_{2}, \lambda\right)
\end{aligned}
$$

for $\lambda \in[0,1]$. Note that $0 \leq y=\lambda \leq 1, p x-k=\lambda\left(p x_{2}-k_{2}\right) \leq \lambda C=C y$, and $k=\lambda k_{2} \leq \lambda u x_{2}=u x$. Finally,

$$
\begin{aligned}
k^{a} & =\left(\lambda k_{2}\right)^{a}=\lambda^{b} k_{2}^{a} \lambda^{a-b}=\left((1-\lambda) 0+\lambda k_{2}^{a / b}\right)^{b} \lambda^{a-b} \\
& \leq\left((1-\lambda) r_{1}+\lambda r_{2}\right)^{b} \lambda^{a-b}=r^{b} y^{a-b} .
\end{aligned}
$$

Thus, $(x, k, r, y) \in S^{\prime}$.

Now, we will show that $S^{\prime} \subseteq \operatorname{conv}(S)$. Consider $(x, k, r, y) \in S^{\prime}$. Note that, if $y \in\{0,1\}$, then $(x, k, r, y) \in S \subseteq \operatorname{conv}(S)$. Thus, assume that $0<y<1$. Then, $(x, k, r, y)$ can be expressed as a convex combination of $(0,0,0,0) \in S$ and $(x / y, k / y, r / y, 1)$ with coefficients $1-\lambda$ and $\lambda=y \in(0,1)$, respectively. As $(x, k, r, y) \in S^{\prime}, p(x / y)-k / y \leq C, k / y \leq$ $u(x / y)$, and

$k^{a} \leq r^{b} y^{a-b} \Rightarrow k^{a / b} \leq r y^{a / b-1} \Rightarrow\left(\frac{k}{y}\right)^{a / b} \leq \frac{r}{y}$.

Consequently, $(x / y, k / y, r / y, 1) \in S$ and $(x, k, r, y) \in \operatorname{conv}(S)$.

Now, we will reformulate constraint (15) using conic quadratic inequalities. As given in BenTal and Nemirovski [4], for a positive integer $l$, and $\varepsilon, \pi_{1}, \ldots, \pi_{2^{l}} \geq 0$

$\varepsilon^{2^{l}} \leq \pi_{1}, \ldots, \pi_{2^{l}}$,

can be represented by using $O\left(2^{l}\right)$ variables and $O\left(2^{l}\right)$ hyperbolic inequalities of the form

$v^{2} \leq w_{1} w_{2}$ 
where $v, w_{1}, w_{2} \geq 0$. Moreover, inequality (17) is conic quadratic representable

$\left\|\left(\begin{array}{c}2 v \\ w_{1}-w_{2}\end{array}\right)\right\| \leq w_{1}+w_{2}$.

Using these results, one can show that for given $t, a \geq b>0$ and $a, b \in \mathbb{Z}_{+}$, inequality (15) can be represented by $O\left(\log _{2}(a)\right)$ variables and conic quadratic constraints of the form (18) [2]. Note that if we fix $y_{t}=1$, then we obtain (14), thus these constraints are also conic quadratic representable. We will refer to the conic quadratic formulations of LS-II and LS-III as CLS-II and CLS-III, respectively.

In CLS-II and CLS-III, for each period $t$, inequalities (14) and (15) are replaced with their conic quadratic representations. Therefore, these formulations are quadratically constrained MIP's (MIQCP) with linear objective functions that can be solved by fast algorithms of commercial MIQCP solvers like IBM ILOG CPLEX. In the next example, we illustrate the generation of conic quadratic constraints.

Example 4.1. Our compression cost for period $t$ is given by $\gamma_{t}\left(k_{t}\right)=\kappa_{t} k_{t}^{a / b}$. We first introduce auxiliary variable $r_{t}$, add inequality $k_{t}^{a / b} \leq r_{t}$ to the formulation and replace $\gamma_{t}\left(k_{t}\right)$ by $\kappa_{t} r_{t}$ in the objective function. Suppose that $a=5$ and $b=2$. Then, for period $t$, we have inequality $k_{t}^{5 / 2} \leq r_{t}$, which can be rewritten as $k_{t}^{5} \leq r_{t}^{2}$. By strengthening the latter inequality, we obtain $k_{t}^{5} \leq r_{t}^{2} y_{t}^{3}$ and it is equivalent to

$k_{t}^{8} \leq r_{t}^{2} y_{t}^{3} k_{t}^{3}$.

This inequality can be expressed with the following four inequalities where three new nonnegative auxiliary variables $w_{1 t}, w_{2 t}$, $w_{3 t} \geq 0$ are introduced:

$w_{1 t}^{2} \leq r_{t} y_{t}$,

$w_{2 t}^{2} \leq y_{t} k_{t}$,

$w_{3 t}^{2} \leq w_{2 t} k_{t}$,

$k_{t}^{2} \leq w_{1 t} w_{3 t}$.

Fig. 1 illustrates the generation of these inequalities.

These constraints can be represented by the following conic quadratic inequalities:

$4 w_{1 t}^{2}+\left(r_{t}-y_{t}\right)^{2} \leq\left(r_{t}+y_{t}\right)^{2}$,

$4 w_{2 t}^{2}+\left(y_{t}-k_{t}\right)^{2} \leq\left(y_{t}+k_{t}\right)^{2}$,

$4 w_{3 t}^{2}+\left(w_{2 t}-k_{t}\right)^{2} \leq\left(w_{2 t}+k_{t}\right)^{2}$,

$4 k_{t}^{2}+\left(w_{1 t}-w_{3 t}\right)^{2} \leq\left(w_{1 t}+w_{3 t}\right)^{2}$.

Consequently, for a given period $t$, each inequality (19) is represented by four conic quadratic inequalities and additional three nonnegative variables $w_{1 t}, w_{2 t}, w_{3 t} \geq 0$. These inequalities can be easily input to a MIQCP solver.

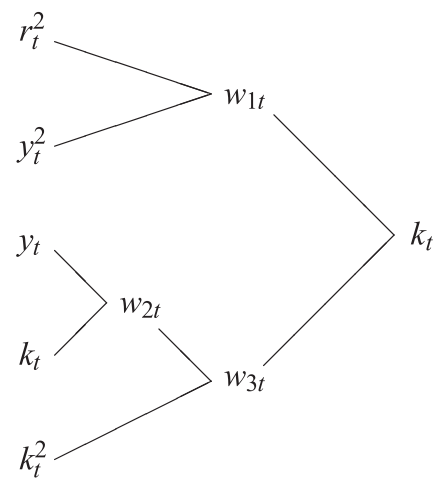

Fig. 1. Illustration of generation of conic quadratic inequalities.

\section{Computational experiments}

In this section, first we will test the effect of strengthening (14) by performing a computational experiment for comparing formulations CLS-II and CLS-III. Then, we will investigate the effect of controllable processing times in terms of cost reduction by comparing optimal costs of the system with and without controllable processing times. In our computational experiments, we consider quadratic and cubic compression cost functions $\gamma_{t}\left(k_{t}\right)=\kappa_{t} k_{t}^{2}$ and $\gamma_{t}\left(k_{t}\right)=\kappa_{t} k_{t}^{3}$. We implement all the formulations in IBM ILOG CPLEX 12.5 and perform the experiments on a $2.4 \mathrm{GHz}$ Intel Core i7 Machine with 16 GB memory running Windows 8.

\subsection{Comparison of formulations}

In the first part of our study, we consider the data sets for $T=50$ periods and with time invariant parameters. Therefore, we delete the subscript $t$ from the parameters. We assume that unit inventory holding cost $(h)$ is 1 , unit production cost $(c)$ is 0 , capacity of a period in terms of time units $(C)$ is 300 , production time without any compression $(p)$ is 1 , maximum possible compression amount $(u)$ for a unit is $30 \%$ of the processing time and coefficient of variation (hereafter $C V$ ) is $10 \%$. We determine the rest of the parameters according to the following values: $\alpha \in\{0.95,0.98\}, q / h \in\{1750,3500$, $7000\}, \kappa / h \in\{0.10,0.30\}, C / \mu p \in\{3,5\}$ and $\mu_{t} \sim U[0.9 \mu, 1.1 \mu]$ for $t=$ $1, \ldots, T$. We set time limit as $2000 \mathrm{~s}$.

Most of the commercial solvers, such as IBM ILOG CPLEX, can solve MIP formulations with a quadratic objective function. Therefore, we also use formulation LS-Q where we keep the quadratic compression cost function in the objective. We note that LS-Q is the same as LS-II except that $\kappa_{t} r_{t}$ is replaced by $\kappa_{t} k_{t}^{2}$ in the objective function, constraints (14) and variables $r_{t}$, for $t=1, \ldots, T$, are removed. We solve LS-Q by CPLEX MIQP. Note that for the quadratic compression cost function, conic reformulations CLS-II and CLS-III are equivalent to LS-II and LS-III, respectively. Thus, performance differences of LS-Q and CLS-II will show the effect of having quadratic terms in the objective function and in the constraints. The effect of proposed conic strengthening can be observed by comparing CLS-II and CLS-III.

Results of this experiment are given in Tables 1 and 4. In these tables, the percentage gap between the continuous relaxation at the root node and the optimal solution (rgap) (root gap, hereafter) and the number of branch-and-bound nodes explored are reported. If the solver is terminated due to the time limit, final gap is given under the column (gap), otherwise solution time is reported (cpu).

Results of this experiment for quadratic compression cost function are given in Table 1 . This table clearly indicates that CLS-III outperforms CLS-II both in terms of root gap and solution time. Note that the root gap of CLS-II is twice as large as of the one of CLS-III for some instances. Moreover, all the instances are solved to optimality in less than $800 \mathrm{~s}$ by CLS-III (average solution time is about 200 s) whereas CLS-II stops with positive gap due to time limit for 10 out of 24 instances. When we examine the results of LS-Q an interesting result is obtained: it can solve an instance within $2 \mathrm{~s}$, whereas for another one it stops with $1 \%$ optimality gap due to time limit. Moreover, LS-Q solves 10 instances in less time than CLS-III, but its solution time seems not so stable. It solves an instance which is solved by CLS-III in about $300 \mathrm{~s}$ in only $4 \mathrm{~s}$. On the other hand, another instance that is solved by CLS-III in less than $40 \mathrm{~s}$ is solved by LS-Q in about $2000 \mathrm{~s}$. When we investigate the instances in detail, we observe that when setup cost increases and capacities become tighter, solution time of LS-Q increases. These results may be related to root gaps and sizes of the formulations. Note that root gap of CLS-II and LS-Q are the same and root gap of CLS-III is better for all of the instances. In Table 2, 
Table 1

Effect of strengthening - quadratic compression cost.

\begin{tabular}{|c|c|c|c|c|c|c|c|c|c|c|c|c|}
\hline \multicolumn{4}{|c|}{ Parameters } & \multicolumn{3}{|l|}{ LS-Q } & \multicolumn{3}{|l|}{ CLS-II } & \multicolumn{3}{|c|}{ CLS-III } \\
\hline$\alpha$ & $q$ & $\kappa$ & $\frac{c}{\mu p}$ & rgap & cpu (gap) & Node \# & rgap & cpu (gap) & Node \# & rgap & cpu (gap) & Node \# \\
\hline \multirow[t]{12}{*}{0.98} & \multirow[t]{4}{*}{1750} & \multirow[t]{2}{*}{10} & 3 & 5.43 & 96 & $2,359,855$ & 5.43 & $(0.11)$ & $23,100,921$ & 3.7 & 473 & $5,787,570$ \\
\hline & & & 5 & 9.35 & 36 & 847,454 & 9.35 & 331 & $5,375,748$ & 6.55 & 71 & 978,035 \\
\hline & & \multirow[t]{2}{*}{30} & 3 & 3.93 & 4 & 102,059 & 3.93 & 473 & $6,561,712$ & 3.37 & 289 & $2,974,113$ \\
\hline & & & 5 & 7.43 & 11 & 274,120 & 7.43 & 166 & $1,827,603$ & 6.3 & 95 & $1,438,351$ \\
\hline & \multirow[t]{4}{*}{3500} & \multirow[t]{2}{*}{10} & 3 & 8.27 & $(0.1)$ & $30,941,006$ & 8.27 & (1.57) & $13,608,874$ & 3.97 & 514 & $4,071,992$ \\
\hline & & & 5 & 11.54 & 46 & $1,036,294$ & 11.54 & 194 & $2,570,270$ & 6.42 & 10 & 127,100 \\
\hline & & \multirow[t]{2}{*}{30} & 3 & 5.49 & 170 & $4,091,687$ & 5.49 & $(0.46)$ & $17,685,610$ & 3.52 & 434 & $3,874,277$ \\
\hline & & & 5 & 9.7 & 37 & 870,979 & 9.7 & 216 & $2,405,941$ & 5.9 & 30 & 355,178 \\
\hline & \multirow[t]{4}{*}{7000} & \multirow[t]{2}{*}{10} & 3 & 9.04 & 1885 & $26,624,840$ & 9.04 & (1.61) & $16,855,483$ & 3.27 & 21 & 246,465 \\
\hline & & & 5 & 12.23 & 29 & 663,867 & 12.23 & 109 & $1,146,528$ & 5.82 & 5 & 55,868 \\
\hline & & \multirow[t]{2}{*}{30} & 3 & 8.87 & $(0.96)$ & $36,503,808$ & 8.87 & (2.38) & $10,696,593$ & 3.04 & 635 & $4,206,533$ \\
\hline & & & 5 & 12.82 & 87 & $1,742,690$ & 12.82 & 217 & $2,522,865$ & 4.86 & 8 & 111,879 \\
\hline \multirow[t]{12}{*}{0.95} & \multirow[t]{4}{*}{1750} & \multirow[t]{2}{*}{10} & 3 & 5.59 & 76 & $1,914,746$ & 5.59 & $(0.11)$ & $17,113,480$ & 3.8 & 573 & $6,126,573$ \\
\hline & & & 5 & 9.34 & 28 & 643,479 & 9.34 & 291 & $3,729,740$ & 6.52 & 67 & 867,485 \\
\hline & & \multirow[t]{2}{*}{30} & 3 & 3.91 & 3 & 82,141 & 3.91 & 700 & $9,037,474$ & 3.34 & 187 & $2,046,082$ \\
\hline & & & 5 & 7.45 & 9 & 240,922 & 7.45 & 130 & $1,467,783$ & 6.32 & 81 & $1,016,680$ \\
\hline & \multirow[t]{4}{*}{3500} & \multirow[t]{2}{*}{10} & 3 & 8.17 & 1954 & $31,230,960$ & 8.17 & $(0.92)$ & $15,831,461$ & 3.87 & 287 & $2,426,309$ \\
\hline & & & 5 & 11.82 & 54 & $1,175,641$ & 11.82 & 191 & $2,418,919$ & 6.66 & 14 & 163,615 \\
\hline & & \multirow[t]{2}{*}{30} & 3 & 5.32 & 140 & $3,276,211$ & 5.32 & 1965 & $13,261,271$ & 3.36 & 233 & $2,195,079$ \\
\hline & & & 5 & 9.54 & 27 & 630,580 & 9.54 & 105 & $1,584,731$ & 5.71 & 25 & 301,468 \\
\hline & \multirow[t]{4}{*}{7000} & \multirow[t]{2}{*}{10} & 3 & 9.12 & 1951 & $28,910,438$ & 9.12 & (1.39) & $18,760,285$ & 3.34 & 31 & 369,543 \\
\hline & & & 5 & 12.25 & 34 & 736,537 & 12.25 & 99 & $1,178,800$ & 5.84 & 2 & 27,418 \\
\hline & & \multirow[t]{2}{*}{30} & 3 & 8.96 & $(1.02)$ & $39,450,904$ & 8.96 & $(2.25)$ & $10,807,958$ & 3.04 & 663 & $5,630,375$ \\
\hline & & & 5 & 12.92 & 137 & $2,942,472$ & 12.92 & 467 & $4,241,231$ & 4.99 & 19 & 231,237 \\
\hline
\end{tabular}

we report the number of variables and constraints of the formulations for quadratic and cubic compression functions. Note that for the quadratic case, LS-Q has the smallest number of constraints and variables and CLS-II and CLS-III have the same number of variables and constraints. What can be observed form these results is the following. Although the number of variables and constraints is increased for conic quadratic reformulation in CLS-II compared to $\mathrm{LS}-\mathrm{Q}$ as gaps on the root nodes are the same for both the formulations, LS-Q performs better than CLS-II. On the other hand, root gap of CLS-III is improved at the expense of increasing model size. Therefore, for relatively easier instances, smaller formulation, as in LS-Q may perform better whereas for the harder ones the formulation with smaller root gaps, as in CLS-III, may be better.

For the cubic compression cost function, we need to add all the conic inequalities. Hyperbolic inequalities, used in the conic reformulations, can be seen in Table 3. Note that the first inequalities used are the same for both the formulations and the second inequality used in CLS-III implies the one used in CLS-II. For the cubic compression cost function, we also consider another strengthened formulation, in which rather than using inequalities $k_{t}^{3} \leq r_{t} y_{t}^{2}$ (given by (15) for $a=3, b=1$ ), we use inequalities $k_{t}^{3} \leq r_{t} y_{t}$, for $t=1, \ldots, T$. This formulation and its conic reformulation will be referred as LS-IV and CLS-IV, respectively. Inequalities used for CLS-IV are also given in Table 3. Note that more variables and hyperbolic inequalities are used for CLS-IV, and the inequalities are different from the inequalities used in CLS-II and CLS-III.

According to the results for cubic compression cost function, given in Table 4, conic strengthening again improves the root gap of CLS-II. However, for this case improvement is not as good as for the quadratic case: for the quadratic compression cost function average root gap reduction is about $4 \%$ ( $40 \%$, relatively), but for the cubic compression cost function it is about $1 \%$ (20\%, relatively). Although root gap for CLS-III is the best, the performance of CLS-IV could be viewed as better since it solves all the instances within the time limit and its average solution time is about $120 \mathrm{~s}$. The difference between CLS-II and CLS-III is not clear for this case: 18 out of 24 instances are solved by both the formulations, and 13 of them are solved in less time by CLS-III. There is one instance that is solved by
Table 2

Number of variables and constraint of the formulations.

\begin{tabular}{llllll}
\hline$a / b$ & \# of & LS-Q & CLS-II & CLS-III & CLS-IV \\
\hline \multirow{3}{*}{2} & Variables & $4 T$ & $5 T$ & $5 T$ & - \\
& Linear constraints & $3 T$ & $3 T$ & $3 T$ & - \\
& Quadratic constraints & - & $T$ & $T$ & - \\
\multirow{3}{*}{3} & Variables & - & $6 T$ & $6 T$ & $7 T$ \\
& Linear constraints & - & $3 T$ & $3 T$ & $3 T$ \\
& Quadratic constraints & - & $2 T$ & $2 T$ & $3 T$
\end{tabular}

-: Not applicable.

Table 3

Hyperbolic inequalities for cubic compression cost function.

\begin{tabular}{lll}
\hline CLS-II & CLS-III & CLS-IV \\
\hline$w_{t}^{2} \leq r_{t} k_{t}$ & $w_{t}^{2} \leq r_{t} k_{t}$ & $w_{t}^{2} \leq r_{t} y_{t}$ \\
$k_{t}^{2} \leq w_{t}$ & $k_{t}^{2} \leq w_{t} y_{t}$ & $v_{t}^{2} \leq k_{t}$ \\
$w_{t} \geq 0$ & $w_{t} \geq 0$ & $k_{t}^{2} \leq w_{t} v_{t}$ \\
& & $w_{t}, v_{t} \geq 0$ \\
\hline
\end{tabular}

CLS-II but not by CLS-III, but three of the instances that cannot be solved by CLS-II are solved by CLS-III. Moreover, if we investigate the results in more detail, we can observe that CLS-III mostly performs better than CLS-II in harder instances (with large setup costs and tighter capacities). The number of variables and constraints for these formulations is also given in Table 2. Note that the number of variables and constraints of CLS-IV are larger than the ones for CLS-II and CLS-III, and the latter two formulations have equal number of variables and constraints. Although the size of CLSIV is larger, the root gap of this formulation is not the best. On the other hand, this formulation performs better in terms of solution times. This situation may be caused by the different types of conic inequalities added to this formulation (Table 2).

Overall, we observed that conic strengthening improves root gaps. This improvement is more definite for the quadratic 
Table 4

Effect of strengthening - cubic compression cost.

\begin{tabular}{|c|c|c|c|c|c|c|c|c|c|c|c|c|}
\hline \multicolumn{4}{|c|}{ Parameters } & \multicolumn{3}{|c|}{ CLS-II } & \multicolumn{3}{|c|}{ CLS-III } & \multicolumn{3}{|c|}{ CLS-IV } \\
\hline$\alpha$ & $q$ & $\kappa$ & $\frac{c}{\mu p}$ & rgap & cpu (gap) & Node \# & rgap & cpu (gap) & Node \# & rgap & cpu (gap) & Node \# \\
\hline \multirow[t]{12}{*}{0.98} & \multirow[t]{4}{*}{1750} & \multirow[t]{2}{*}{10} & 3 & 3.91 & 838 & $5,078,538$ & 3.56 & 1341 & $6,749,843$ & 3.69 & 33 & 121,618 \\
\hline & & & 5 & 7.09 & 225 & $2,250,718$ & 6.42 & 329 & $2,445,704$ & 6.62 & 111 & 681,670 \\
\hline & & \multirow[t]{2}{*}{30} & 3 & 3.86 & 765 & $4,589,460$ & 3.66 & $(0.01)$ & $9,577,594$ & 3.73 & 31 & 125,007 \\
\hline & & & 5 & 7.17 & 550 & $4,163,002$ & 6.78 & 575 & $4,485,975$ & 6.9 & 195 & 876,346 \\
\hline & \multirow[t]{4}{*}{3500} & \multirow[t]{2}{*}{10} & 3 & 4.13 & 1305 & $7,609,046$ & 3.3 & 815 & $3,392,463$ & 3.6 & 159 & 396,016 \\
\hline & & & 5 & 7.6 & 285 & $2,424,222$ & 6.01 & 157 & $1,400,172$ & 6.49 & 50 & 222,179 \\
\hline & & \multirow[t]{2}{*}{30} & 3 & 3.65 & 785 & $4,396,585$ & 3.17 & 416 & $3,148,587$ & 3.34 & 18 & 56,223 \\
\hline & & & 5 & 6.67 & 84 & 463,807 & 5.72 & 78 & 486,854 & 6.01 & 28 & 137,715 \\
\hline & \multirow[t]{4}{*}{7000} & \multirow[t]{2}{*}{10} & 3 & 4.43 & $(0.73)$ & $6,272,523$ & 2.67 & 525 & $2,301,886$ & 3.3 & 137 & 378,028 \\
\hline & & & 5 & 8.15 & 126 & 968,464 & 4.61 & 52 & 354,709 & 5.66 & 29 & 93,946 \\
\hline & & \multirow[t]{2}{*}{30} & 3 & 3.62 & $(0.19)$ & $7,861,026$ & 2.6 & 770 & $4,104,475$ & 2.97 & 23 & 69,675 \\
\hline & & & 5 & 6.43 & 67 & 571,358 & 4.37 & 26 & 178,464 & 4.99 & 28 & 138,135 \\
\hline \multirow[t]{12}{*}{0.95} & \multirow[t]{4}{*}{1750} & \multirow[t]{2}{*}{10} & 3 & 4.01 & 1105 & $6,042,570$ & 3.66 & 884 & $5,961,144$ & 3.79 & 46 & 140,596 \\
\hline & & & 5 & 7.49 & 650 & $4,722,629$ & 6.83 & 484 & $4,625,674$ & 7.03 & 192 & 897,207 \\
\hline & & \multirow[t]{2}{*}{30} & 3 & 3.96 & $(0.26)$ & $7,772,856$ & 3.76 & $(0.55)$ & $16,234,025$ & 3.83 & 43 & 191,853 \\
\hline & & & 5 & 7.21 & 573 & $4,411,070$ & 6.82 & 523 & $3,540,305$ & 6.94 & 173 & 878,045 \\
\hline & \multirow[t]{4}{*}{3500} & \multirow[t]{2}{*}{10} & 3 & 4.03 & 1425 & $6,803,350$ & 3.21 & 331 & $1,813,792$ & 3.5 & 43 & 109,090 \\
\hline & & & 5 & 7.66 & 181 & $1,403,050$ & 6.06 & 253 & $1,429,436$ & 6.54 & 59 & 281,301 \\
\hline & & \multirow[t]{2}{*}{30} & 3 & 3.52 & 476 & $2,942,293$ & 3.04 & 282 & $1,702,281$ & 3.21 & 36 & 102,870 \\
\hline & & & 5 & 6.83 & 70 & 546,947 & 5.9 & 121 & 774,107 & 6.18 & 44 & 179,112 \\
\hline & \multirow[t]{4}{*}{7000} & \multirow[t]{2}{*}{10} & 3 & 4.92 & 2000 & $7,669,462$ & 3.11 & $(0.14)$ & $8,461,099$ & 3.75 & 1231 & $2,744,415$ \\
\hline & & & 5 & 8.37 & 254 & $1,837,744$ & 4.82 & 110 & 873,418 & 5.87 & 96 & 373,115 \\
\hline & & \multirow[t]{2}{*}{30} & 3 & 3.86 & $(0.84)$ & $6,647,400$ & 2.82 & 918 & $4,570,382$ & 3.19 & 183 & 569,582 \\
\hline & & & 5 & 6.42 & 54 & 382,068 & 4.38 & 31 & 160,493 & 5 & 20 & 87,244 \\
\hline
\end{tabular}

compression cost function, since CLS-III outperforms CLS-II for this case. But for the cubic compression cost function, CLS-IV, in which more conic inequalities are used, outperforms CLS-III, for our instances. In summary, by utilizing second-order cone programming, we could solve the relatively practical sizes of stochastic capacitated lot sizing problem with a nonlinear compression cost function in a reasonable computation time instead of relying on a heuristic approach.

\subsection{Effect of controllable processing times}

Controlling the capacity of the system can be a beneficial tool to hedge against demand uncertainty. For this purpose, in this section, we report the results of several experiments to show the benefits of controlling processing times under different uncertainty/cost/capacity settings. In order to achieve this, we will compare the optimal costs for the problem with and without controllable processing times, which will be called as LS-C and LS, respectively, and report the cost reduction. In this part, we again assume that all the parameters are time invariant, and the compression cost function is quadratic or cubic. We consider instances with $T=20, h=1, c=0$, $C=300$ and $p=1$. The rest of the parameters is generated according to the ratios given in Table 5 . We consider different capacity and demand scenarios by considering different $C / \mu p$ and $\beta$ values. For example, for $\beta=0.5$, and $C / \mu p=5$, mean demand of period $t$ is generated as $\mu_{t} \sim U[30,90]$ since $\mu=60$ for this setting. Thus, when $\beta$ is smaller, mean demand of each period becomes close to each other and when it increases it is possible to have fluctuating mean demand. We also consider different demand variability levels by considering different coefficient of variation settings. Note that according to Table 5, there are 972 different parameter settings for both quadratic and cubic compression cost functions. Moreover, we generated five replications for each setting, thus we generated 4860 randomly generated problem instances for both the functions. We summarize the results of this experiment in Tables 6-9. As all the instances are solved to optimality in less than one second, we do not report solution times in this section. In order to see the effect of controllable processing times under different scenarios, we
Table 5

Experimental design factors and their settings.

\begin{tabular}{lllccr}
\hline \multirow{2}{*}{ Factor } & Explanation & \# of levels & \multicolumn{4}{l}{ Factor settings } \\
\cline { 3 - 5 } & & & 1 & 2 & 3 \\
\cline { 3 - 6 } & & 2 & 0.95 & 0.99 \\
$\alpha$ & Service level & 3 & 1750 & 3500 & 7000 \\
$q$ & Setup cost & 3 & 0.01 & 0.5 & 1 \\
$\kappa$ & Compression cost coefficient & 3 & 5 & 10 & 20 \\
$C /(\mu p)$ & Avg. capacity tightness & 3 & 10 & 30 & 50 \\
$u$ & Max. possible compression $(\%)$ & 3 & 0.1 & 0.5 & - \\
$\beta$ & $\mu_{t} \sim U[(1-\beta) \mu,(1+\beta) \mu]$ & 2 & 10 & 30 & 50 \\
$C V$ & Coeff. of variation $(\%)$ & 3 & & & \\
\hline
\end{tabular}

report the improvements for different combinations of parameters. In these tables, the value on the first row of each cell represents the average percentage cost reduction $(\Delta)$ for given parameter settings, and the maximum percentage cost reduction $\left(\Delta_{\max }\right)$ obtained over all instances with this setting is given in the second row.

\subsubsection{Effect of setup costs}

We obtain an overall $6.54 \%$ average cost improvement for the quadratic compression cost function. Table 6 gives the percentage improvements for different service level $\alpha$, setup cost $q$, coefficient of variation $C V$ and capacity values. We first observe that $\Delta$ increases as set up cost increases. While the set up cost increases, compressing the processing times and reducing the number of production periods becomes more valuable. When we examine the difference between the number of production periods for LS and LS-C, we see that the average reduction in the number of production periods is about $0.45,0.63$ and 0.73 for $q=1750,3500,7000$, respectively. For these setup cost values, average percentage cost reduction is $1.48,6.15$ and 11.99 , respectively, and $\Delta_{\max }$ may be as high as 30\% when setup cost is high.

When we investigate the results in detail, we observe that all the improvements are not due to reduction in the number of production periods. In about 688 (out of 4860) instances, though the number of 
Table 6

Service level vs. setup cost vs. capacity vs. CV (quadratic).

\begin{tabular}{|c|c|c|c|c|c|c|c|c|c|c|c|c|c|}
\hline \multirow[t]{4}{*}{$C / \mu p$} & \multirow[t]{4}{*}{$\alpha$} & \multicolumn{12}{|c|}{ Setup cost } \\
\hline & & \multicolumn{4}{|l|}{1750} & \multicolumn{4}{|l|}{3500} & \multicolumn{4}{|l|}{7000} \\
\hline & & \multicolumn{3}{|l|}{$\mathrm{CV}$} & \multirow[b]{2}{*}{ Avg. } & \multicolumn{3}{|l|}{$\mathrm{CV}$} & \multirow[b]{2}{*}{ Avg. } & \multicolumn{3}{|l|}{$\mathrm{CV}$} & \multirow[b]{2}{*}{ Avg. } \\
\hline & & 10 & 30 & 50 & & 10 & 30 & 50 & & 10 & 30 & 50 & \\
\hline \multirow[t]{5}{*}{5} & \multirow[t]{2}{*}{0.99} & 2.41 & 1.6 & 2.15 & \multirow[t]{2}{*}{2.05} & 6.44 & 4.07 & 4.86 & \multirow[t]{2}{*}{5.12} & 10.89 & 7.34 & 8.18 & \multirow[t]{2}{*}{8.8} \\
\hline & & 6.63 & 7.18 & 7.41 & & 15.64 & 17.43 & 17.38 & & 24.18 & 28.75 & 28.62 & \\
\hline & \multirow[t]{2}{*}{0.95} & 2.96 & 1.52 & 1.5 & \multirow[t]{2}{*}{1.99} & 6.99 & 4.02 & 3.74 & \multirow[t]{2}{*}{4.92} & 11.39 & 8.09 & 6.6 & \multirow[t]{2}{*}{8.69} \\
\hline & & 6.95 & 6.61 & 6.99 & & 15.95 & 15.62 & 16.91 & & 24.43 & 24.16 & 28.29 & \\
\hline & Avg. & 2.69 & 1.56 & 1.83 & 2.03 & 6.72 & 4.05 & 4.3 & 5.02 & 11.14 & 7.72 & 7.39 & 8.75 \\
\hline \multirow[t]{5}{*}{10} & \multirow[t]{2}{*}{0.99} & 2.62 & 1.08 & 0.62 & \multirow[t]{2}{*}{1.44} & 8.78 & 3.77 & 2.34 & \multirow[t]{2}{*}{4.96} & 15.62 & 8.64 & 4.54 & \multirow[t]{2}{*}{9.6} \\
\hline & & 5.69 & 5.87 & 4.79 & & 13.47 & 13.43 & 12.29 & & 20.62 & 20.5 & 19.53 & \\
\hline & \multirow[t]{2}{*}{0.95} & 3.22 & 1.73 & 0.89 & \multirow[t]{2}{*}{1.95} & 9.83 & 6.69 & 3.23 & \multirow[t]{2}{*}{6.58} & 17.2 & 14.08 & 7.26 & \multirow[t]{2}{*}{12.85} \\
\hline & & 5.83 & 6.3 & 5.59 & & 13.62 & 13.9 & 13.13 & & 20.75 & 20.9 & 20.25 & \\
\hline & Avg. & 2.92 & 1.41 & 0.76 & 1.7 & 9.31 & 5.23 & 2.79 & 5.78 & 16.41 & 11.36 & 5.9 & 11.22 \\
\hline \multirow[t]{5}{*}{20} & 0.99 & 0.42 & 1.02 & 0.26 & \multirow[t]{2}{*}{0.57} & 4.5 & 11.36 & 3.95 & 6.6 & 8.41 & 23.47 & 12 & 14.63 \\
\hline & & 3.94 & 4.96 & 3.65 & & 17.53 & 18.18 & 16.7 & & 29.58 & 29.95 & 28.7 & \\
\hline & 0.95 & 0.59 & 1.47 & 0.64 & 0.9 & 4.76 & 12.74 & 8.68 & 8.73 & 8.59 & 24.06 & 19.45 & 17.37 \\
\hline & & 4.14 & 3.84 & 4.63 & & 17.77 & 17.65 & 17.79 & & 29.78 & 29.77 & 29.62 & \\
\hline & Avg. & 0.51 & 1.25 & 0.45 & 0.74 & 4.63 & 12.05 & 6.32 & 7.67 & 8.5 & 23.77 & 15.73 & 16 \\
\hline Avg. & & 2.04 & 1.4 & 1.01 & 1.48 & 6.88 & 7.11 & 4.47 & 6.15 & 12.02 & 14.28 & 9.67 & 11.99 \\
\hline
\end{tabular}

Table 7

Setup cost vs. $\kappa$ (quadratic).

\begin{tabular}{ccccc}
\hline$q$ & $\kappa$ & & \multirow{2}{*}{ Avg. } \\
\cline { 2 - 4 } & 0.01 & 0.5 & 1 & \\
\hline \multirow{2}{*}{1750} & 2.92 & 0.9 & 0.62 & 1.48 \\
& 7.41 & 5.81 & 5.78 & \\
3500 & 9.73 & 4.96 & 3.77 & 6.15 \\
7000 & 18.18 & 17.52 & 17.3 & \\
& 16.27 & 10.8 & 8.89 & 11.99 \\
Avg. & 29.95 & 29.65 & 29.54 & \\
& 9.64 & 5.55 & 4.43 & 6.54 \\
\hline
\end{tabular}

Table 8

Capacity vs. mean demand variability vs. max. possible compression (quadratic).

\begin{tabular}{|c|c|c|c|c|c|c|c|c|}
\hline \multirow[t]{4}{*}{$\frac{c}{\mu p}$} & \multicolumn{8}{|l|}{$\beta$} \\
\hline & \multicolumn{4}{|l|}{10} & \multicolumn{4}{|l|}{50} \\
\hline & \multicolumn{3}{|l|}{$u$} & \multirow[b]{2}{*}{ Avg. } & \multicolumn{3}{|l|}{$u$} & \multirow[b]{2}{*}{ Avg. } \\
\hline & 10 & 30 & 50 & & 10 & 30 & 50 & \\
\hline \multirow[t]{2}{*}{5} & 3.66 & 6.08 & 7.18 & \multirow[t]{2}{*}{5.64} & 3.08 & 5.15 & 6.43 & \multirow[t]{2}{*}{4.89} \\
\hline & 12.98 & 24.43 & 28.62 & & 13.1 & 23.75 & 28.75 & \\
\hline \multirow[t]{2}{*}{10} & 5.47 & 8.07 & 8.07 & \multirow[t]{2}{*}{7.2} & 3.35 & 6.16 & 6.26 & \multirow[t]{2}{*}{5.26} \\
\hline & 20.75 & 20.75 & 20.75 & & 20.9 & 20.9 & 20.9 & \\
\hline \multirow[t]{2}{*}{20} & 6.39 & 8.7 & 8.7 & \multirow[t]{2}{*}{7.93} & 5.91 & 9.54 & 9.54 & \multirow[t]{2}{*}{8.33} \\
\hline & 29.77 & 29.77 & 29.77 & & 29.95 & 29.95 & 29.95 & \\
\hline Avg. & 5.17 & 7.62 & 7.98 & 6.92 & 4.11 & 6.95 & 7.41 & 6.16 \\
\hline
\end{tabular}

production periods is the same for LS and LS-C, cost reduction is obtained by compressing the processing times and reducing the total inventory holding cost. However, since setup cost is the dominant one among the other cost terms, the average improvement for these instances is about $0.16 \%$ (maximum is $1.77 \%$ ).

\subsubsection{Effect of capacity and demand parameters}

Parameters $\alpha, C V$ and $C / \mu p$ affect the difference between capacity and modified demand $\hat{d}$. Since we assume that the capacity $C$ and the unit processing time $p$ are constant, $C / \mu p$ increases only when the mean demand $\mu$ decreases. Thus, capacity compared to the modified demand increases with $C / \mu p$. When the service level $\alpha$ increases, $Z_{\alpha}$ and consequently, the modified demand parameter $\hat{d}$ increases. Similarly, when $C V$ increases $\sigma$ increases and again, $\hat{d}$ increases. Thus, for larger $\alpha$ or $C V$ values it is possible to have tighter capacities relative to the demand. Note that when capacities are large enough to satisfy the demand, which is possible when $\alpha$ and $C V$ are smaller and $C / \mu p$ is larger, compressing the processing times may not be a preferred option. For example, controllable processing times have no advantage if the system is uncapacitated. On the other hand, when capacities are tight which is possible for larger $\alpha$ and $C V$ values and smaller $C / \mu p$, even though the processing times are compressed, it may not be possible to obtain a better solution or the improvement may be small relative to the total cost. Note that in this case, more compression should be done in order to reduce the number of production periods and as compression cost is convex, compression may not be beneficial anymore. Therefore, controllable processing times are more beneficial when capacities are medium sized relative to the modified demand.

Results given in Table 6 confirm the observations explained above. For example, for $C / \mu p=5$ or $10, \Delta$ is maximum when $C V=10$ and if $C / \mu p$ is increased to $20, \Delta$ is larger for $C V=30$.

$\alpha$ and the coefficient of variation have the same effect on the modified demand, but according to Table $6, \Delta$ is more affected by the changes in the coefficient of variation. Note that the changes in $C V$ affect the modified demand in larger amounts and this is the reason of larger changes of $\Delta$ with respect to $C V$.

When we investigate the results in more detail, we observe that as the capacity increases, the total cost of LS decreases, in general. Therefore, even though the cost reduction due to controllable processing times is the same for different capacity settings, as $\Delta$ indicates the percentage cost improvement, $\Delta$ may be higher for larger capacity settings. An example of this situation is observed for $C V=10$ and $C / \mu p=5$ or 10 .

To sum up, according to Table 6 , we can conclude that controllable processing times are more beneficial when setup costs are high and the difference between the capacities and the modified demand is medium sized. 
Table 9

Service level vs. setup cost vs. capacity vs. CV (cubic).

\begin{tabular}{|c|c|c|c|c|c|c|c|c|c|c|c|c|c|}
\hline \multirow[t]{4}{*}{$C / \mu p$} & \multirow[t]{4}{*}{$\alpha$} & \multicolumn{12}{|c|}{ Setup cost } \\
\hline & & \multicolumn{4}{|l|}{1750} & \multicolumn{4}{|l|}{3500} & \multicolumn{4}{|l|}{7000} \\
\hline & & \multicolumn{3}{|l|}{$\mathrm{CV}$} & \multirow[b]{2}{*}{ Avg. } & \multicolumn{3}{|l|}{$\mathrm{CV}$} & \multirow[b]{2}{*}{ Avg. } & \multicolumn{3}{|l|}{$\mathrm{CV}$} & \multirow[b]{2}{*}{ Avg. } \\
\hline & & 10 & 30 & 50 & & 10 & 30 & 50 & & 10 & 30 & 50 & \\
\hline \multirow[t]{5}{*}{5} & \multirow[t]{2}{*}{0.99} & 0.85 & 0.56 & 1.02 & \multirow[t]{2}{*}{0.81} & 2.53 & 1.45 & 2.33 & \multirow[t]{2}{*}{2.1} & 4.9 & 3.16 & 3.79 & \multirow[t]{2}{*}{3.95} \\
\hline & & 4.85 & 3.92 & 3.98 & & 8.94 & 8.12 & 7.32 & & 12.82 & 12.35 & 10.55 & \\
\hline & \multirow[t]{2}{*}{0.95} & 1.59 & 0.31 & 0.37 & \multirow[t]{2}{*}{0.76} & 3.66 & 1.27 & 0.77 & \multirow[t]{2}{*}{1.9} & 6.46 & 2.76 & 1.7 & \multirow[t]{2}{*}{3.64} \\
\hline & & 5.08 & 4.7 & 3.65 & & 9.14 & 8.82 & 7.06 & & 12.97 & 12.74 & 11.62 & \\
\hline & Avg. & 1.22 & 0.44 & 0.7 & 0.79 & 3.1 & 1.36 & 1.55 & 2 & 5.68 & 2.96 & 2.75 & 3.8 \\
\hline \multirow[t]{5}{*}{10} & \multirow[t]{2}{*}{0.99} & 1.26 & 0.16 & 0.01 & \multirow[t]{2}{*}{0.48} & 4.31 & 0.88 & 0.03 & \multirow[t]{2}{*}{1.74} & 9.5 & 2.63 & 0.28 & \multirow[t]{2}{*}{4.14} \\
\hline & & 5.68 & 4.77 & 0.1 & & 13.46 & 12.63 & 0.99 & & 20.61 & 19.98 & 12.13 & \\
\hline & \multirow[t]{2}{*}{0.95} & 1.64 & 0.58 & 0.1 & \multirow[t]{2}{*}{0.77} & 6.72 & 3.16 & 0.44 & \multirow[t]{2}{*}{3.44} & 12.69 & 6.13 & 1.98 & \multirow[t]{2}{*}{6.93} \\
\hline & & 5.83 & 6.07 & 2.93 & & 13.62 & 13.74 & 11.19 & & 20.75 & 20.79 & 18.99 & \\
\hline & Avg. & 1.45 & 0.37 & 0.06 & 0.63 & 5.52 & 2.02 & 0.24 & 2.59 & 11.1 & 4.38 & 1.13 & 5.54 \\
\hline \multirow[t]{6}{*}{20} & \multirow[t]{2}{*}{0.99} & 0.17 & 0.34 & 0 & 0.17 & 2.27 & 4.33 & 0.62 & 2.41 & 5.67 & 10.24 & 3.32 & 6.41 \\
\hline & & 3.64 & 4.73 & 0 & & 17.32 & 18.02 & 10.95 & & 29.45 & 29.85 & 25.02 & \\
\hline & 0.95 & 0.22 & 0.78 & 0.16 & 0.39 & 3.47 & 7.28 & 2.92 & 4.56 & 6.54 & 16.37 & 6.77 & 9.89 \\
\hline & & 4.06 & 3.81 & 3.67 & & 17.71 & 17.63 & 17.11 & & 29.75 & 29.75 & 29.19 & \\
\hline & Avg. & 0.2 & 0.56 & 0.08 & 0.28 & 2.87 & 5.81 & 1.77 & 3.48 & 6.11 & 13.31 & 5.05 & 8.16 \\
\hline & Avg. & 0.96 & 0.46 & 0.28 & 0.56 & 3.83 & 3.06 & 1.19 & 2.69 & 7.63 & 6.88 & 2.97 & 5.83 \\
\hline
\end{tabular}

\subsubsection{Other parameters}

Table 7 shows the percentage improvements for setup cost and compression cost coefficient $\kappa$. When $\kappa$ increases, as it is expected, the compression cost increases and consequently, the cost reduction that can be obtained by compressing the processing times decreases. But note that when setup cost increases, it is more beneficial to compress the processing times, even with larger $\kappa$, and it is possible to have a reduction of $29 \%$.

In Table 8 , the percentage improvements for different capacity levels, maximum possible compression amounts $(u)$ and mean demand scenarios are given. As expected, if $u$ increases, the cost difference between LS and LS-C, and consequently, $\Delta$ may increase. If capacities are large enough, larger $u$ values may not change the optimal production plan. Note that $\Delta$ and $\Delta_{\max }$ are the same for different $u$ values when capacities are large enough. Moreover, average cost and number of production periods differences are the same for these settings. On the other hand, when capacity is tighter, increasing the maximum possible compression amount increases the improvement.

According to Table 8 , the effect of $\beta$ on $\Delta$ is relatively small. More improvement is observed when capacities are looser and mean demand fluctuates more or capacities are tighter and mean demand is stable. When we examine the results in detail, we obtain that when capacity is large enough and mean demand is stable, without reducing processing times an optimal production plan can be observed. Moreover, we note that in none of the test instances with $C / \mu p=20, C V=10$ and $\beta=0.1$, a cost reduction is observed. But this setting is an extreme case as capacity is large enough and with only one production period all the necessary demand is produced.

\subsubsection{Cubic compression cost function}

In Table 9, results for the cubic compression cost function are given. In this table, we report the percentage improvements for different service level $\alpha$, setup cost $q$, coefficient of variation $C V$ and capacity values. The average improvement is $3.03 \%$. When the compression cost function is changed from quadratic to cubic, $\Delta$ decreases since compression becomes more expensive (when $k_{t} \geq 1$ ). Although the average improvement decreases, behavior of
$\Delta$ is very similar to one of the quadratic cases. Note that for some settings $\Delta_{\max }$ may be as high as the one for quadratic compression cost function, but also for some parameter combinations it may be zero. As similar results are obtained for this case, we do not report the impact of other problem parameters in detail.

As a result of our computational experiments, we can say that taking processing times as constant increases system costs and even with small compressions it is possible to have less costly production plans. Although the controllable processing times provide cost improvements in most of the randomly generated problem instances, we observe largest improvements when setup costs are high, compression costs are low and capacities are medium sized compared to the modified demands.

\section{Conclusions}

In this study, we consider the stochastic capacitated lot sizing problem with controllable processing times where compression cost function is a convex function of the compression amount. To the best of our knowledge, this problem is not studied before. We formulate this problem using SOCP, strengthen the formulation and solve the proposed formulations using a commercial solver.

As a result of our computational experiments, we observe that conic strengthening performs quite well, and it improves gaps in the root node. Consequently, we can solve realistic size problems in a reasonable computation time using an exact approach. Moreover, these formulations maybe further improved by adding new valid inequalities in a future research.

Although controllable processing times may be applicable to many real life production and inventory systems, processing times are assumed as constant in the classical lot sizing problems. In the second part of our computational experiments, we show that this assumption causes higher system costs. We observe that controllable processing times are more valuable when the system has medium sized capacities and larger setup costs. By relaxing the fixed processing time assumption and by utilizing the recent advances in second-order cone programming, we could decrease the overall production costs significantly. Although we explore these results for the static uncertainty strategy under $\alpha$ service 
level constraints, these results are also applicable to the deterministic lot sizing problem. As a future research direction, it is possible to consider the same problem under different settings like considering different uncertainty strategies, joint chance constraints or using multistage stochastic programming approach to deal with different scenarios.

\section{Acknowledgments}

The authors thank the area editor, the associate editor, and anonymous referees for their constructive comments and suggestions that significantly improved this paper. This research is supported by TUBITAK Project no .112M220. The research of the second author is supported by the Turkish Academy of Sciences.

\section{References}

[1] Ahmed S, King AJ, Parija G. A multi-stage stochastic integer programming approach for capacity expansion under uncertainty. Journal of Global Optimization 2003;26:3-24

[2] Aktürk MS, Atamtürk A, Gürel S. A strong conic quadratic reformulation for machine-job assignment with controllable processing times. Operations Research Letters 2009;37(3):187-91.

[3] Atamtürk A, Hochbaum DS. Capacity acquisition, subcontracting, and lot sizing. Management Science 2001:47(8):1081-100.

[4] Ben-Tal A, Nemirovski A. Lectures on modern convex optimization: analysis, algorithms, and engineering applications. Society for Industrial and Applied Mathematics, Philadelphia, PA; 2001.

[5] Bookbinder JH, Tan J-Y. Strategies for probabilistic lot-sizing problem with service-level constraints. Management Science 1988;34(9):1096-108.

[6] Chen FY, Krass D. Inventory models with minimal service level constraints. European Journal of Operational Research 2001;134(1):120-40.

[7] L Croxton K, Gendron B, Magnanti TL. A comparison of mixed-integer programming models for nonconvex piecewise linear cost minimization problems. Management Science 2003;49(9):1268-73.

[8] Florian M, Lenstra JK, Rinnooy Kan AHG. Deterministic production planning: algorithms and complexity. Management Science 1980;26(7):669-79.

[9] Gürel S, Körpeoğlu E, Aktürk MS. An anticipative scheduling approach with controllable processing times. Computers \& Operations Research 2010;37 (6):1002-13.

[10] Helber S, Sahling F, Schimmelpfeng K. Dynamic capacitated lot sizing with random demand and dynamic safety stocks. OR Spectrum 2013;35:75-105.

[11] Jansen K, Mastrolilli M. Approximation schemes for parallel machine scheduling problems with controllable processing times. Computers \& Operations Research 2004;31(10):1565-81.

[12] Jeang A. Simultaneous determination of production lot size and process parameters under process deterioration and process breakdown. Omega 2012;40:774-81.

[13] Karimi B, Fatemi Ghomi SMT, Wilson JM. The capacitated lot sizing problem: a review of models and algorithms. Omega 2003;31(5):365-78.

[14] Kayan RK, Aktürk MS. A new bounding mechanism for the CNC machine scheduling problems with controllable processing times. European Journal of Operational Research 2005;167(3):624-43.

[15] Koca E, Yaman H, Aktürk MS. Lot sizing with piecewise concave production costs. INFORMS Journal on Computing 2014;26(4):767-79.

[16] Laserre JB, Bes C, Roubellat F. The stochastic discrete dynamic lot size problem: an open-loop solution. Operations Research 1985;33(3):684-9.
[17] Luss H. Operations research and capacity expansion problems: a survey Operations Research 1982;30(5):907-47.

[18] Merzifonluoğlu Y, Geunes J, Romeijn H. Integrated capacity, and production planning with subcontracting and overtime options. Naval Research Logistics 2007;54(4):433-47.

[19] Nahmias S. Production and operations analysis. Boston: McGraw-Hill/Irwin; 2005.

[20] Özen U, Doğru MK, Tarım SA. Static-dynamic uncertainty strategy for a singleitem stochastic inventory control problem. Omega 2012;40:348-57.

[21] Pochet Y, Wolsey LA. Production planning by mixed integer programming New York, NY: Springer; 2006.

[22] Rajagopalan S, Swaminathan JM. A coordinated production planning mode with capacity expansion and inventory management. Management Science 2001;47(11):1562-80.

[23] Robinson P, Narayanan A, Sahin F. Coordinated deterministic dynamic demand lot-sizing problem: a review of models and algorithms. Omega 2009;37(1):3-15.

[24] Rossi R, Kılıc OA. Tarım SA. Piecewise linear approximations for the staticdynamic uncertainty strategy in stochastic lot-sizing. Omega 2015;50:126-40.

[25] Shabtay D, Kaspi M. Minimizing the total weighted flow time in a single machine with controllable processing times. Computers \& Operations Research 2004;31(13):2279-89.

[26] Shabtay D, Kaspi M. Parallel machine scheduling with a convex resource consumption function. European Journal of Operational Research 2006;173 (1):92-107.

[27] Shabtay D, Steiner G. A survey of scheduling with controllable processing times. Discrete Applied Mathematics 2007;155(13):1643-66.

[28] Shabtay D, Steiner G. Single machine batch scheduling to minimize total completion time and resource consumption costs. Journal of Scheduling 2007;10(4-5):255-61.

[29] Shaw DX, Wagelmans APM. An algorithm for single-item capacitated economic lot sizing with piecewise linear production costs and general holding costs. Management Science 1998;44(6):831-8.

[30] Silver E. Inventory control under a probabilistic time-varying, demand pattern. AIIE Transactions 1978;10(4):371-9.

[31] Sox C. Dynamic lot sizing problem with demand and non-stationary demand Operations Research Letters 1997;20:155-64.

[32] Tarım SA, Kingsman BG. The stochastic dynamic production/inventory lotsizing problem with service-level constraints. International Journal of Production Economics 2004;88:105-19.

[33] Tempelmeier H. A column generation heuristic for dynamic capacitated lot sizing with random demand under a fill rate constraint. Omega 2011;39:627-33.

[34] Tunç H, Kılıç O, Tarım SA, Ekșioğlu B. The cost of using stationary inventory policies when demand is non-stationary. Omega 2011;39(4):410-5.

[35] Tunç H, Kılıç OA, Tarım SA, Ekşioğlu B. A simple approach for assessing the cost of system nervousness. International Journal of Production Economics 2013;141(2):619-25.

[36] Tunç H, Kılıç OA, Tarım SA, Ekşioğlu B. A reformulation for the stochastic lo sizing problem with service-level constraints. Operations Research Letters 2014:42(2):161-5.

[37] Türkcan A, Aktürk MS, Storer RH. Predictive/reactive scheduling with controllable processing times and earliness-tardiness penalties. IIE Transactions 2009:41(12):1080-95.

[38] Vargas V. An optimal solution for the stochastic version of the Wagner-Whitin dynamic lot-size model. European Journal of Operational Research 2009;198:447-51.

[39] Vickson RG. Two single machine sequencing problems involving controllable job processing times. AIIE Transactions 1980;12(3):258-62.

[40] Wagner HM, Whitin TM. Dynamic version of the economic lot size model. Management Science 1958;5(1):89-96.

[41] Yan C, Liao Y, Banerjee A. Multi-product lot scheduling with backordering and shelf-life constraints. Omega 2013;41:510-6. 